\title{
Can We Remember Future Actions yet Forget the Last Two Minutes? Study in Transient Global Amnesia
}

\author{
Mathieu Hainselin ${ }^{1}$, Peggy Quinette ${ }^{1}$, Béatrice Desgranges ${ }^{1}$, \\ Olivier Martinaud $^{2}$, Didier Hannequin ${ }^{2}$, Vincent de La Sayette ${ }^{1}$, \\ Fausto Viader ${ }^{1}$, and Francis Eustache ${ }^{1}$
}

\begin{abstract}
Transient global amnesia (TGA) is a clinical syndrome characterized by the abrupt onset of a massive episodic memory deficit that spares other cognitive functions. If the anterograde dimension is known to be impaired in TGA, researchers have yet to investigate prospective memory (PM) — which involves remembering to perform an intended action at some point in the future-in this syndrome. Furthermore, as executive functions are thought to be spared in this syndrome, TGA provides an opportunity to examine the impact of a massive "pure" memory impairment on PM. We assessed 38 patients with a newly designed protocol that distinguished between the prospective (remembering to do something at the appropriate time) and retrospective (remembering what has to be done) components of PM. Moreover,
\end{abstract}

\section{INTRODUCTION}

Transient global amnesia (TGA) is a neurological syndrome that occurs in middle age and lasts up to $24 \mathrm{hr}$ but whose etiology remains elusive. It is characterized by the sudden onset of massive anterograde amnesia, usually accompanied by variable retrograde amnesia and repetitive questioning. The memory deficit chiefly affects episodic memory, and the executive functions (inhibition, dual task performance, updating and shifting mechanisms) are considered to be largely spared (Quinette et al., 2003) or with slight impairment (Jäger, Bazner, Kliegel, Szabo, \& Hennerici, 2009). Whereas anterograde and retrograde episodic memory in TGA have been extensively studied, to our knowledge, there has not been any research on any aspect of the memory oriented in the future in TGA. And yet, given that episodic memory is currently defined as the ability to mentally travel into both the past and the future (Eustache \& Desgranges, 2008; Tulving, 2002; Wheeler, Stuss, \& Tulving, 1997), it seems important, both at the clinical and theoretical levels, to find out whether these patients suffering from massive amnesia can nonetheless

${ }^{1}$ INSERM U923, EPHE, Université de Caen Basse-Normandie, Centre Hospitalier Universitaire de Caen, ${ }^{2}$ INSERM U614, Centre Hospitalier Universitaire de Rouen we investigated episodic memory with an anterograde memory task and assessed executive functions, anxiety and mood, as well as their links with PM. We demonstrated that PM is impaired during TGA, with a greater deficit for the retrospective component than for the prospective component. Furthermore, we highlighted a strong link between these two components. Anterograde episodic memory impairments were correlated with retrospective component deficits in TGA patients, although we were able to confirm that executive functions are globally spared. We discuss this pattern of results within the theoretical framework of PM, putting forward new arguments in favor of the idea that PM deficits can occur mainly because of a massive anterograde memory deficit. The clinical consequences of PM impairment in TGA are examined.

remember to perform actions in the future, in other words, to assess their prospective memory (PM).

PM refers to the ability to remember to perform an intended action at some point in the future (Einstein, McDaniel, Richardson, Guynn, \& Cunfer, 1995). When this action involves the detection of an external cue (e.g., delivering a message to a colleague when we next bump into him or her), it is called an event-based PM task; when this action involves checking the time (e.g., going to the hairdresser's at 5 p.m.), it is called a time-based PM task (Einstein \& McDaniel, 1990).

PM has been studied in various samples of patients with memory disorders, in particular mild cognitive impairment (Blanco-Campal, Coen, Lawlor, Walsh, \& Burke, 2009; Karantzoulis, Troyer, \& Rich, 2009) and Alzheimer's disease (Kinsella, Ong, Storey, Wallace, \& Hester, 2007; Jones, Livner, \& Backman, 2006). Thompson, Henry, Rendell, Withall, and Brodaty (2010), who compared these two populations, have suggested that the PM impairment in these diseases may reflect executive difficulties, rather than genuine episodic memory difficulties, as the patients' performances on executive tasks were also poor and were found to be correlated with the PM task scores. These results raise the question of the involvement of executive functions in PM functioning. There is also a debate about PM in normal aging, for whereas 
some studies have reported poorer PM performances for older participants (Gonneaud, Kalpouzos, Bon, et al., 2011), others have not (Uttl, 2008). For their part, Martin, Kliegel, and McDaniel (2003) claimed that "frontal/executive functioning is an important predictor for PM performance." Beyond the executive functioning debate, the implication of anterograde memory in PM is still not clear, although these two aspects of memory are important research topics (Henry, MacLeod, Phillips, \& Crawford, 2004).

One possible explanation for these heterogeneous results is that some authors do not regard PM as a divisible entity. Einstein and McDaniel (1990) put forward the idea that PM is supported by two related components: a prospective component (ProCom), which refers to remembering when to do something (i.e., at the appropriate time), and a retrospective component (RetCom), which refers to remembering what to do at that time. However, these two components are difficult to study separately in two different but comparable tasks. Previous studies have encountered this very difficulty, for although they have been able to assess the RetCom by giving participants a clue to compensate for any ProCom deficit (Livner, Laukka, Karlsson, \& Backman, 2009; Adda, Castro, Alem-Mar e Silva, de Manreza, \& Kashiara, 2008; Costa, Peppe, Caltagirone, \& Carlesimo, 2008; Carlesimo, Casadio, \& Caltagirone, 2004; Cohen, Dixon, Lindsay, \& Masson, 2003), but these paradigms did not assess the ProCom directly.

Functional neuroimaging studies during PM tasks have described a pattern of activation involving the frontal areas, notably Brodmann's area 10 (Burgess, Scott, \& Frith, 2003; Burgess, Quayle, \& Frith, 2001; Okuda et al., 1998), as well as temporal neural structures (Reynolds, West, \& Braver, 2009; den Ouden, Frith, Frith, \& Blakemore, 2005). This involvement of frontal and temporal structures in PM reflects findings relating to the "prospective brain" (Schacter, Addis, \& Buckner, 2007), a core brain system that mediates episodic memory for the past and simulates future events (projection in the future, which is another form of memory oriented in the future). This system encompasses the medial prefrontal regions, posterior regions in the medial and lateral parietal cortex, lateral temporal cortex, and medial temporal lobe. The role played by the frontal and temporal lobes in PM has been confirmed in a study conducted by Umeda, Naqumo, and Kato (2006), based on an investigation of two patients during rehabilitation. This study suggested that the ProCom is supported by the frontal lobes, which are involved in executive functions, whereas the RetCom is supported by the temporal lobes, which underpin anterograde episodic memory.

An interesting way of exploring the hypothesis of a possible distinction between the ProCom and RetCom further is to investigate PM in a sudden-onset, temporary, and massive amnesic syndrome without major executive functions impairment, such as TGA. Hence, the first aim of the present study was to assess PM in a group of TGA patients, characterized by pure episodic memory impairment, without any residual ability or cognitive re- organization such as that seen in Alzheimer's disease or other permanent amnesias. The second objective was to establish whether a distinction can be made between the prospective and RetCom of PM, using a specially designed protocol. Moreover, we planned to examine the anterograde dimension of episodic memory, the executive functions, the three subcomponents of working memory (phonological loop, visual sketchpad, and central executive), the binding process, and anxiety and mood to control for their putative effects on both components of PM. By means of correlations, we wanted to study how these neuropsychological, anxiety, and mood factors might influence PM functioning in TGA. By so doing, we hoped to gain a better clinical understanding of TGA and its symptoms (anxiety, repetitive questioning) and a clearer theoretical view of PM and its links with executive functions.

\section{METHODS}

\section{Participants}

Thirty-eight TGA patients, admitted to the emergency departments of Caen and Rouen University Hospitals between January 2008 and January 2010, took part in the study. Descriptive data are reported in Table 1 . All patients met the standard clinical criteria for the diagnosis of TGA published by Hodges and Warlow (1990) and underwent a neurological examination, CT scan, and/or EEG, all of which were normal except for five of them (three EEG revealed minor abnormalities without epileptic features and two CT scans revealed moderate atrophy). Seventeen patients were examined during the acute phase, 10 during the periacute phase, and 11 on the day after the beginning of amnesia. To estimate the end of the acute phase, which is always difficult to gauge because the recovery process is so gradual (Quinette, Guillery-Girard, Dayan, et al., 2006), we used several indicators (Noël et al., 2008): When patients displayed neither temporal disorientation nor repetitive questioning but still had moderate memory impairment $(z$ score $>-1.65$, but lower than during the acute phase) attested by their scores on two story recall tests-the Wechsler Memory Scale (Wechsler, 1969) and the "Batterie d'efficience mnésique" (Signoret, 1991) — they were included in the periacute phase group. To check that the patients' memory did not significantly improve during the administration of the protocol and ensure that they were included in the appropriate group, the story recall tests were administered both at the beginning and at the end of the protocol; no patient showed a significant improvement between the two evaluations. The patients evaluated more than $24 \mathrm{hr}$ after the onset of the symptoms were included in the "day after" group. These different groups (acute, periacute, and day after) were constituted according to when the patients were admitted to the emergency department.

The mean duration of the episode in these 38 patients was approximately $4.5 \mathrm{hr}(S D=0 \mathrm{hr} 33 \mathrm{~min})$. For all 
Table 1. Clinical and Demographic Characteristics of Individuals with TGA and Controls

\begin{tabular}{|c|c|c|c|c|}
\hline & $\begin{array}{l}\text { Acute Phase } \\
\quad(n=17)\end{array}$ & $\begin{array}{l}\text { Periacute Phase } \\
\quad(n=10)\end{array}$ & $\begin{array}{l}\text { Day After } \\
(n=11)\end{array}$ & $\begin{array}{l}\text { Healthy Controls } \\
\quad(n=14)\end{array}$ \\
\hline Gender: women/men & $15 / 2$ & $8 / 2$ & $8 / 3$ & $9 / 5$ \\
\hline Age in years: mean $(S D)$ & $65.88(6.99)$ & $61.20(6.11)$ & $66.54(8.66)$ & $63.07(9.84)$ \\
\hline $\begin{array}{l}\text { Level of education in years: } \\
\text { mean }(S D)\end{array}$ & $12.06(2.79)$ & $10.8(2.04)$ & $12.63(4.06)$ & $11.29(5.20)$ \\
\hline $\begin{array}{l}\text { Duration of the acute phase of } \\
\text { TGA in hours: mean }(S D)\end{array}$ & $7.32(4.52)$ & $2.78(1.88)$ & $4.56(2.34)$ & - \\
\hline $\begin{array}{l}\text { Time of examination after onset of } \\
\text { TGA in hours: mean }(S D)\end{array}$ & $3.6(1.3)$ & $7.6(5.2)$ & $21.4(7.3)$ & - \\
\hline No. of recurrences & 3 (second episode) & 1 (second episode) & 0 & - \\
\hline No. of precipitants & & & & - \\
\hline Stressful event & 4 & 2 & 1 & \\
\hline Physical effort & 4 & 2 & 3 & \\
\hline Intercourse & 2 & 1 & 1 & \\
\hline Water contact & 1 & 2 & 1 & \\
\hline No precipitant & 6 & 3 & 5 & \\
\hline No. of risk factors & & & & - \\
\hline High blood pressure & 13 & 6 & 7 & \\
\hline Hypercholesterolaemia & 6 & 2 & 2 & \\
\hline Diabetes & 3 & 0 & 1 & \\
\hline Migraine & 6 & 4 & 3 & \\
\hline Depression & 2 & 1 & 2 & \\
\hline
\end{tabular}

of them, we looked for the presence of a previous episode and risk factors reported in previous TGA research, which might provide information concerning the etiology of the episodes (Quinette, Guillery-Girard, Dayan, et al., 2006).

\section{Neuropsychological Assessment}

A neuropsychological and neurological follow-up examination was carried out approximately 2 months later (mean interval $=70.84$ days, $S D=8.23$ days) to rule out any other cognitive impairment. At that point (follow-up group), no patient had any major memory impairment, except for lacunar amnesia for the episode, which is common in this syndrome. However, a slight defect in memory has sometimes been found, and the absence of impairment here might be partly because of a ceiling effect. A control group consisted of 14 healthy people, matched with the TGA patients by age and level of education, as detailed in Table 1. All subjects gave their informed consent to the study, which was approved by the local ethics committee.

The neuropsychological protocol assessed PM, anterograde memory, executive functions, working memory, anxiety, and mood. Because of the specific nature of TGA, it was modular and could be applied to the patients in the form of short sequences interleaved with medical examinations.

Finally, to verify the integrity of general cognitive functions, patients and controls underwent an assessment of orientation and general knowledge. We also used matching figures, semantic categorization (Mattis, 1976), and assessed visuoconstructive abilities (Signoret et al., 1989).

\section{Prospective Memory}

We adopted an event-based task paradigm, as a time-based one would not have been possible, because of the massive anterograde amnesia that characterizes TGA (in a preliminary experiment, we found that, as patients forgot what had occurred within the previous $2 \mathrm{~min}$, it was impossible for them to remember to check for the time and perform the appropriate action, even a simple one like picking up a pencil after $1 \mathrm{~min}$ ). We administered two distinct eventbased tasks, together with a similar ongoing task, to assess the ProCom and RetCom separately, as far as possible, using a direct measure. In the literature, the ProCom has often been assessed with an indirect measure, only relevant when both components are preserved: When the participants fail to carry out the prospective task, the 
experimenter asks them if there is something they are meant to do (i.e., he or she compensates for a possible ProCom impairment by helping them to remember when to do something, and ProCom is never assessed independently; Livner et al., 2009; Adda et al., 2008; Costa et al., 2008; Carlesimo et al., 2004; Cohen et al., 2003). This only allows ProCom integrity to be assessed if the RetCom is preserved, too (i.e., the participant performs the correct prospective task at the appropriate time). Here, we measured the number of correct responses for both PM tasks (ProCom and RetCom) as well as the ongoing task. The design is presented in Figure 1 and detailed below.

\section{Ongoing Task (Similar Design for Both Tasks)}

This task was inspired by the devise by d'Ydewalle, Bouckaert, and Brunfaut (2001) and consisted of 240 mental arithmetic problems as the ongoing activity. A series of simple addition problems was presented, and the participants were asked to press the D key if the addition was correct and the L key if it was not. The key responses were counterbalanced across participants. The latter were told to stay focused throughout this task and to answer the questions as quickly as possible without making any mistakes.

\section{ProCom Task}

Ten specific items were randomly inserted amongst the 240 mental arithmetic sums. The participants were asked to press the $\mathrm{Y}$ key whenever an addition was printed in red, regardless of whether or not that addition was correct. To maximize the involvement of the ProCom, we played down the involvement of the RetCom (what patients had to do) with only one possible response for the patient who just had to remember when, and not what, something had to be done.

\section{RetCom Task}

As for the ProCom task, 10 specific items were randomly inserted among another set of 240 mental arithmetic sums. The participants were asked to press the star key (a star sticker was put on the T key) when the addition was printed in blue and the flower key (a flower sticker was put on the $\mathrm{U}$ key) when the addition was printed in green. A response was only deemed to be correct when the participants pressed the correct key. They were told that a message would indicate an imminent color change just before the preceding addition. This particular procedure was designed to minimize the ProCom, so that when the
Figure 1. The ProCom (top) and RetCom (bottom) experimental tasks. Arrows indicate the correct answers on the keyboard for each condition. The "True" button corresponds to the D key, and the "False" button corresponds to the L key (this was counterbalanced between the subjects). The black button in the ProCom task corresponds to the Y key. In the RetCom task, the flower and the star buttons correspond to the $\mathrm{T}$ and $\mathrm{U}$ keys, respectively (see text for full description of the task).

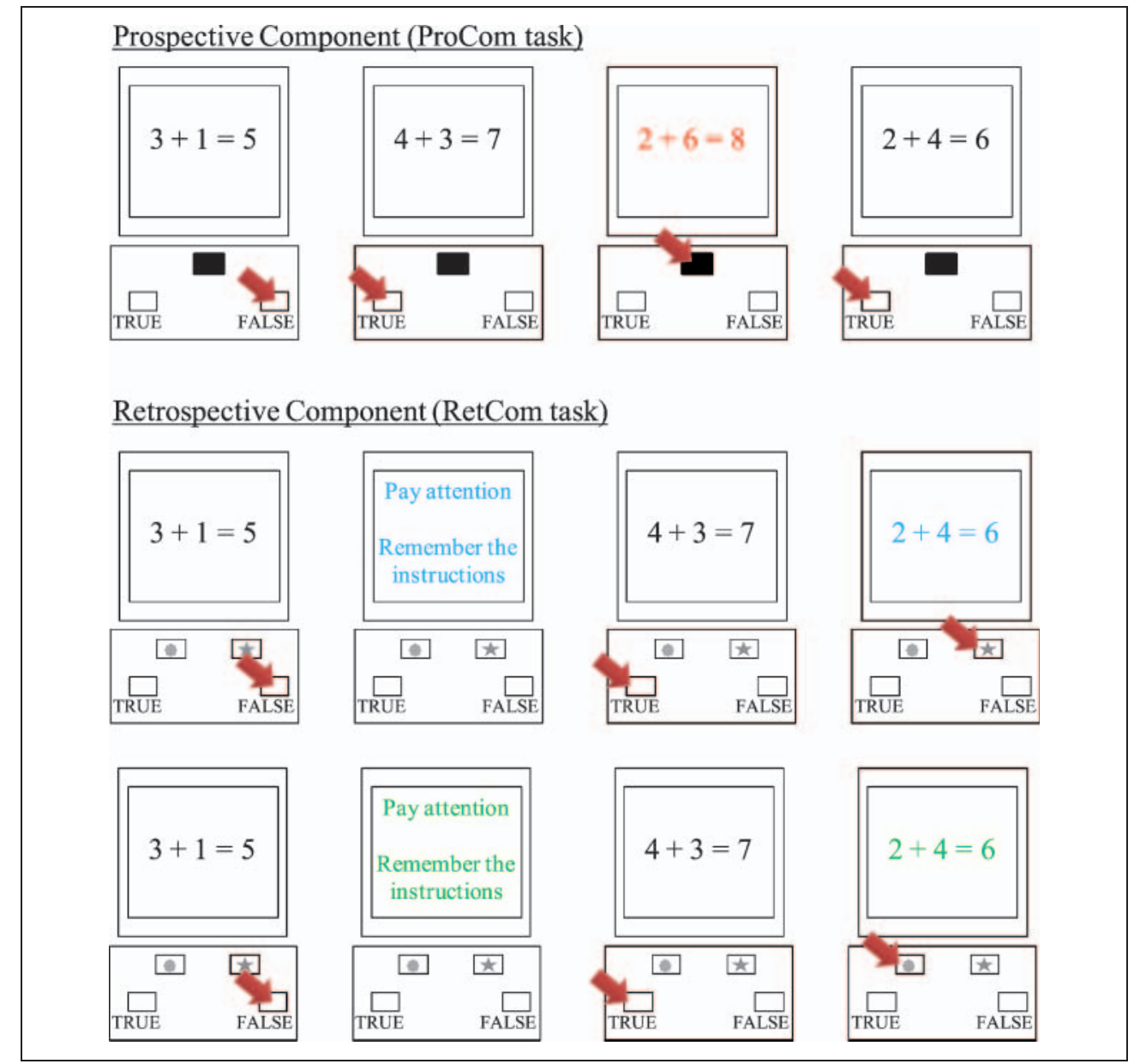


addition was in blue or green, they only had to remember what they had to do (RetCom).

Before starting the PM task, we made sure that patients had completely understood the instructions. After each task, which takes approximately $10 \mathrm{~min}$, participants were quizzed about the initial instructions. Moreover, we compared the first five and last five prospective items to control the PM accuracy throughout PM tasks.

\section{Anterograde Memory Task}

The task used to evaluate anterograde memory was the Encoding-Storage-Retrieval (ESR) test, derived from Grober and Buschke's (1987) procedure. It has been used and described in several previous studies of TGA (Quinette, Guillery-Girard, Noël, et al., 2006; Quinette et al., 2003; Guillery et al., 2000, 2001, 2002; Eustache et al., 1999). The procedure involves the semantic processing of lists of 16 words belonging to 16 different semantic categories, the effectiveness of which is verified by immediate cued recall every two words. Retrieval is assessed by testing free recall and recognition of the first word in each of the 16 lists. We included an $\mathrm{R} / \mathrm{K}$ (remember/know) paradigm in this task to assess the participants' subjective experience during the retrieval process. A "Remember" (R) response corresponded to autonoetic consciousness and the participants' reliving of the event, whereas a "Know" (K) response corresponded to noetic consciousness and was based on a feeling of familiarity. Furthermore, participants could give a "Guess" (G) response when they were not sure of their answer. These responses were all converted into percentages of correct answers.

\section{Executive Functions and Working Memory}

We tested two executive functions (inhibition and shifting) using the Stroop Test and the Trail Making Test (TMT; Godefroy, 2008). The categorical fluency task (taken from the Mattis Dementia Rating Scale; Mattis, 1976) was also administered.

The working memory assessment included three tasks used to explore the three components of Baddeley's (2000) theoretical framework: The forward digit span task (Wechsler, 1991) for the phonological loop, the forward visuospatial span (Wechsler, 1991) for the visuospatial sketchpad, and the letter-number sequence task (Wechsler, 1997) for the central executive.

The processes of binding and maintaining multimodal information were assessed using a working memory binding task and a multimodal span task, respectively, described in a previous study of TGA (Quinette, Guillery-Girard, Noël, et al., 2006). The former consists in matching four consonants with four locations marked by four crosses on a $5 \times 4$ grid, according to their color. As before, we calculated two scores: an accuracy score, taking omissions and false alarms into account, and a processing score, taking failures to respond into account, which may have reflected the fact that pa- tients did not have enough time either to bind the information (i.e., to create a representation in working memory during the presentation) or to retrieve the integrated representation. The multimodal span task consisted in recalling strings of letters placed in an array (Quinette, Guillery-Girard, Noël, et al., 2006). In this task, unlike the binding task, the association (letter and localization) is already done. We took the longest series the patients could remember into account.

\section{Anxiety and Mood Assessments}

Anxiety was assessed by means of the State-Trait Anxiety Inventory (STAI), the first part of which concerns state anxiety and the second part concerns the trait anxiety (Spielberger, 1983). Mood state was evaluated by means of a French adaptation of the Beck Depression Inventory (BDI; Beck, Rial, \& Rickets, 1974) and the BefindlichkeitsSkala adjective mood scale (Von Zerssen, Koeller, \& Rey, 1970). For all these tests, a high score indicates a high level of anxiety or a negative mood.

\section{Statistical Methodology}

The statistical analysis of the PM performances was carried out using a repeated measures ANOVA, with Group (acute phase, periacute phase, day after, healthy controls) as the between-participant factor and PM component (ProCom and RetCom) as the within-participant factor. The first five versus last five prospective items scores were analyzed with a nonparametric $\chi^{2}$. The remaining data were analyzed using a one-way ANOVA, with Group as a betweenparticipant factor. Post hoc Tukey tests were used to carry out paired comparisons.

To determine the link between the two components of PM and the influence of cognitive and psychological factors on patients' PM performances, we established correlations between (1) the ProCom and RetCom scores and (2) these two scores and scores on the cognitive tests and anxiety and mood scales, for which a significant difference was found between patients and controls during TGA. To increase the statistical power in the correlation analyses, we included all the patients with an episodic impairment (acute and periacute phases) in a single group of 27 individuals. The 11 patients from the "day after" group were not included in these analyses, as they did not differ from the control group on the anterograde memory tests.

\section{RESULTS}

The detailed scores are presented in the Appendix.

\section{Prospective Memory}

We found a significant main effect of Group, $F(3,48)=$ 12.89, $p<.0001$, and Component, $F(1,48)=70.94, p<$ .0001 , and a Group $\times$ Component interaction, $F(3,48)=$ 9.69, $p=.054$. Results are detailed in Figure 2 . 


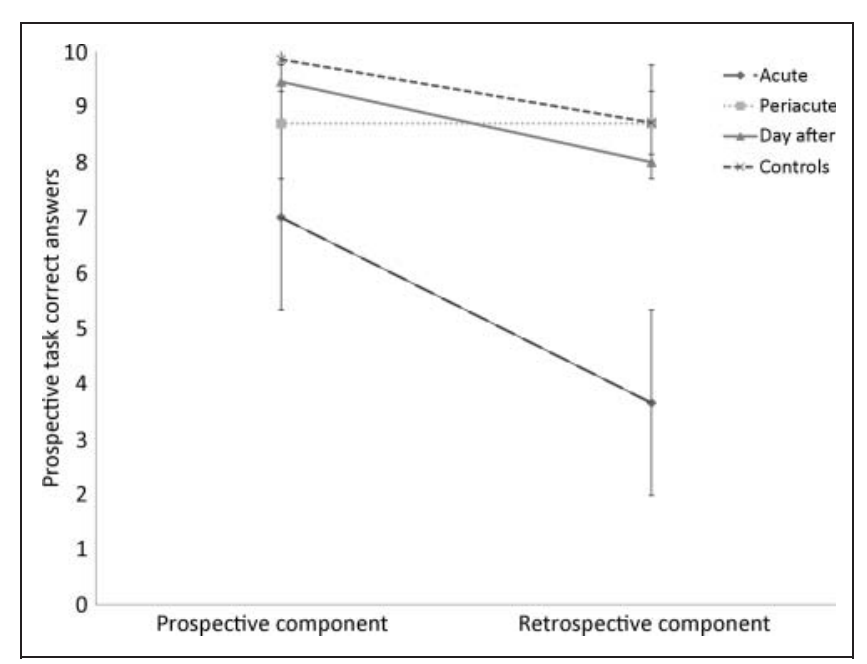

Figure 2. Results of the ProCom and RetCom assessments in TGA patients and healthy controls. Acute-phase patients presented greater impairment for the RetCom than for the ProCom. They performed more poorly than controls for both the ProCom and the RetCom. They also performed more poorly than the patients in the periacute phase and day after groups, but only for the RetCom.

A post hoc analysis revealed that, for the ProCom, acutephase patients performed more poorly than healthy controls. No significant difference was found between the other groups. For the RetCom, acute-phase patients performed more poorly than all the other groups (periacute phase, day after, healthy controls). No difference was found between these other groups. When we compared the two components, the only difference we found was in the acute-phase patients who displayed a greater impairment of the RetCom than of the ProCom.

There was no effect of Group on response time concerning the arithmetic items in the ProCom, $F(3,48)=$ $1.58, p=.206$, and the RetCom tasks, $F(3,48)=1.77$, $p=.164$, neither concerning the prospective items, in the ProCom, $F(3,48)=0.37, p=.774$, and the RetCom tasks, $F(3,48)=0.81, p=.494$.

We found a significant main effect of Group for the instructions quiz about the ProCom, $F(3,48)=4.81, p=$ .005 , and the RetCom tasks, $F(3,48)=14.01, p<.0001$. Post hoc analyses revealed that acute-phase patients remembered significantly fewer instructions than patients assessed the day after the episode and the healthy controls for the ProCom task; they also performed poorer than the three other groups for the RetCom task.

For acute-phase patients, the prospective items accuracy was not significantly different between the first five and the last five items in the ProCom $\left(\chi^{2}=0.077 ; p=.178\right)$ and in the $\operatorname{RetCom}\left(\chi^{2}=1.815 ; p=.193\right)$ tasks.

\section{Anterograde Memory}

As expected, a significant group effect was found for the ESR immediate cued recall, $F(3,48)=11.104, p<.0001$, free re- call, $F(3,48)=18.070, p<.0001$, and recognition tasks, $F(3,48)=11.120, p<.0001$. Post hoc analyses revealed that acute-phase patients performed significantly more poorly than the other three groups whereas patients examined during the periacute phase performed more poorly than the healthy controls, but only in the free recall condition.

An analysis of the $\mathrm{R} / \mathrm{K} / \mathrm{G}$ paradigm results revealed a significant effect of Group on the R, $F(3,48)=64.0125$, $p<.0001$, and $\mathrm{K}$ scores, $F(3,48)=6.615, p=.0008$, whereas no difference was found concerning the $G$ one, $F(3,48)=0.816, p=.4910$. Post hoc Tukey tests showed that the $\mathrm{R}$ scores of patients examined during the acute and periacute phases were lower than those of patients assessed the day after the episode and the healthy controls. Moreover, the acute-phase patients gave fewer R answers than those in the periacute phase. Post hoc analyses showed that the periacute patients gave more $\mathrm{K}$ answers than individuals in the day after and healthy control groups. No other significant differences were observed between the groups for the R, K, or $\mathrm{G}$ answers.

\section{Executive Functions and Working Memory}

There was no effect of Group on time, $F(3,48)=0.0142$, $p=.6978$, or number of errors, $F(3,48)=0.430, p=$ .7322 , in the Stroop interference test. Similarly, scores on Part B of the TMT failed to reveal any difference between groups, whether for time, $F(3,48)=0.466, p=.707528$, or for numbers of errors, $F(3,48)=0.721, p=.5445$.

We did, however, find a significant effect of Group on the numbers of correct responses, $F(3,48)=7.079, p=$ .0005 , and perseverative errors, $F(3,48)=3.760, p=$ .0167 , in the categorical fluency task. Post hoc analyses revealed that patients examined during the acute phase produced significantly fewer correct responses and more perseverative errors than healthy controls.

No statistical difference between the four groups was found for performances on the forward digit span, $F(3$, $48)=0.593, p=.6221$, or letter-number tasks, $F(3,48)=$ $2.576, p=.0647$. However, a group effect was found for the forward visuospatial span, $F(3,48)=2.993, p=.0399$, which was due, according to post hoc analyses, to the poorer performances of patients assessed the day after, compared with acute-phase patients (this could partly be attributed to fatigue after spending a day or a night in hospital). No significant difference was found between acuteand periacute-phase patients' performances or between any of the patient groups and the controls.

We found no effect of Group for the multimodal span task, $F(3,48)=2.576, p=.0647$, or for the accuracy score of the binding task, $F(3,48)=0.727, p=.5411$. There was, however, a significant effect of Group for the processing score, $F(3,48)=6.626, p=.0008$, which took failure to respond into account. Post hoc analyses revealed that acute-phase patients had lower performances than the other three groups, which did not differ significantly from each other in this comparison. 


\section{Anxiety and Mood Assessments}

No significant effect of Group was found for the scores on trait-STAI, $F(3,48)=1.402, p=.2537$, or the BDI, $F(3$, $48)=0.2624, p=.8521$, although we did note a significant difference between scores on the state-STAI, $F(3,48)=$ $25.270, p<.0001$, and the Bf-S, $F(3,48)=7.070, p=$ .0005 . Post hoc analyses showed that acute-phase patients scored higher than every other group on the state-STAI scale whereas periacute phase patients scored higher than controls on this measure. The acute-phase patients also scored higher than controls on the Bf-S.

\section{Correlations between PM, Anterograde Memory, and Anxiety and Mood Scores}

The two PM components were positively correlated with each other $(r=0.61, p<.01)$. We found a significant positive correlation between the ProCom score and the binding (processing) score. There were positive correlations between the RetCom score and the ESR immediate cued recall, recognition, and $\mathrm{R}$ percentage scores, as well as with the binding (processing) score. We also found negative correlations between the RetCom score and both the number of perseverative errors in the fluency task and the state-STAI score. All the correlations are reported in Table 2.

\section{DISCUSSION}

The present study demonstrates that TGA patients present a PM impairment. This is the first time that a PM deficit has been uncovered in a population of patients with

Table 2. Correlations between PM, Anxiety, and Mood Scales and Neuropsychological Scores in Patients during Acute and Periacute Stages ( $r$ Values)

\begin{tabular}{lcc}
\hline & ProCom & RetCom \\
\hline ESR immediate cued recall & +0.37 & $+0.60 * *$ \\
ESR free recall & +0.33 & +0.31 \\
ESR recognition & +0.38 & $+0.53 * *$ \\
ESR \%R answer & +0.33 & $+0.53 * *$ \\
ESR \%K answer & +0.21 & +0.25 \\
Fluency task (perseverative errors) & -0.35 & $-0.39 *$ \\
Binding (processing score) & $+0.41 *$ & $+0.60 *$ \\
State-STAI & -0.28 & $-0.43 *$ \\
Adjective mood scale & -0.31 & -0.18 \\
\hline \%R answers = percentage of "Remember" answers, \%K answers $=$ \\
percentage of "Know" answers. \\
$* p<.05$.
\end{tabular}

a pure episodic memory deficit. This was made possible by the use of a novel event-based paradigm, which enabled us to distinguish between the two components of PM (RetCom and ProCom) and measure the potential impact of anterograde memory on each of them. We discuss below the implications of these results at both the theoretical and clinical levels.

The first aim of our study was to assess PM in TGA. Like anterograde memory, PM is impaired in TGA. The correlations we found between the anterograde memory and PM scores (discussed separately below) suggest that memory for past events and memory for actions to be performed in the future share a common basis. We may also think that they lie on a continuum, in accordance with the "mental time travel" concept proposed by Tulving (2002). Recent research has highlighted a common network for remembering the past and simulating future events (projection into the future), comprising the pFC (Northoff \& Bermpohl, 2004), posterior cingulate cortex (Szpunar, Chan, \& McDermott, 2009), and medial-temporal lobe-including the hippocampus (Viard et al., 2011; Eichenbaum, 2001). This network closely resembles the one implicated in PM (McDaniel \& Einstein, 2010; West \& Krompinger, 2005). This connection between PM and the simulation of future events has already been postulated by Schacter, Addis, and Buckner (2008), in a review of cognitive and neural patterns, but needs to be investigated further. This common network for remembering the past and memory for actions to be performed is also comparable to the regions supposed to be involved in memory impairment in TGA (Bartsch et al., 2006; Guillery et al., 2002). In this perspective, we may assume that the neural perturbations during TGA underlying the anterograde memory impairment can be responsible for the PM impairment as well. In the same way, the absence of massive frontal perturbation during TGA can explain the general preservation of executive functions. This is also discussed below in a neuropsychological perspective.

Moreover, in our study, PM followed the same dynamic course of recovery as anterograde memory, with better performances for both of them as the TGA receded (see Guillery-Girard et al., 2004, for similar results for anterograde and retrograde memory). This result reinforces the hypothesis of a common basis for memory for past events and actions in the future.

The second objective of our study was to distinguish between the ProCom and RetCom. To this end, we implemented a specially designed paradigm that allowed us to gain direct and independent measures of each component, using two simple tasks. We then assessed the links between these two components of PM and different cognitive functions in TGA. We demonstrated that PM is impaired during the acute stage of TGA, and this deficit was greater for the RetCom than for the ProCom. However, this interpretation might be partly because of a possible ceiling effect for controls, as these tasks were adapted for TGA patients, often present in PM studies (Uttl, 2008). Regarding the instructions quiz results, we can ask if 
acute-phase patients performed poorly in the PM tasks just because they forgot the instructions; however, two arguments counter this hypothesis. First, patients did not explicitly recall all the instructions after the tasks but were able to give some piece of it. Second, during the task, just after failing from pressing the correct button after a prospective items (including the very last one), some patients said they forgot to press on the flower button when the addition was printed in green. With this last evidence, put in the perspective of the lack of explicit instructions recall, we suggest that patients are still able to perform a task until they are interrupted although they cannot remember it—or the instructions-after. This is consistent with TGA patients' ability to perform different tasks during a neuropsychological evaluation, although their anterograde memory is impaired. Anyway, taken together, these results and the close correlation between the two components of PM suggest that the massive impairment of the RetCom may influence the ProCom. Hence, it is surely difficult to remember that we have something to do if we cannot remember what that something is. Similarly, in everyday life, we cannot worry about keeping an appointment if we have completely forgotten that we have one in the first place. Although the influence of the ProCom on the RetCom is intuitively obvious (we cannot perform the correct action at the appropriate moment if we forget to perform it) and has been described in previous research, this is the first time, to our knowledge, that the opposite influence has been evoked.

Concerning the ProCom, the only correlation we found was with the binding (processing) score, previously found to be lower for TGA patients (Quinette, Guillery-Girard, Noël, et al., 2006). These results concerning the binding task, used to assess the episodic buffer, are interesting, as the scores were correlated with both components of PM. This seems to reflect the importance of the binding process when it comes to associating the cue with the action to be performed and the point at which it needs to be performed (Wang, Dew, \& Giovanello, 2010). The binding process is also supposed to be important during the retrieval of the association between the intended action and the prospective cue. If many researches considered that the frontal system was the only involved, recent findings highlighted the role of hippocampal region (McDaniel \& Einstein, 2010). This is consistent with the binding process impairment and the early encoding deficit for some TGA patients (Quinette, Guillery-Girard, Noël, et al., 2006) and the results in this study. This aspect of PM will deserve specific extended study in future researches.

Concerning the RetCom, its scores correlated positively with performances on many of the measures of anterograde memory (ESR), supporting the hypothesis that anterograde memory plays a key role in the RetCom (Umeda et al., 2006), as patients with massive amnesia have difficulty remembering what they have to do in the future. The significant correlation between the $\mathrm{R}$ and RetCom scores suggests that the correct answers given by the pa- tients were not because of chance. Moreover, we found a negative correlation between the RetCom and state anxiety scores (although no correlation with mood), consistent with previous results in PM (Kliegel \& Jager, 2006) and TGA concerning the impact of anxiety on episodic memory (Noël et al., 2008). Stress is also supposed to have an impact on hippocampus in TGA (Sedlaczek et al., 2004); moreover, it is, among others, a possible explanation for verbal leitmotifs (discussed above). Anxiety can also interfere with the retrieval of the action to be performed, consistent with a retrieval deficit previously highlighted in TGA (Eustache et al., 1999). This is supported by the poor performance of patients on the fluency task and its correlation with the RetCom task, indicating a deficit in accessing information in memory. Moreover, even if there is no significant correlation in this study, the anterograde memory deficit might also be implicated in the fluency task performance, also evoked in medial-temporal lobe amnesia (Greenberg, Keane, Ryan, \& Verfaellie, 2009).

On the basis of our results and the literature, we have developed a summary figure of PM (Figure 3) featuring its two components (ProCom and RetCom). This figure assumes that anterograde memory plays a key role in the RetCom and, indirectly, in the ProCom, given the strong link between these two components. Executive functions and working memory, thought to play an important role in complex tasks and when the prospective clue is not salient, as well as in time-based or high-demand tasks (Basso, Ferrari, \& Palladino, 2010; Martin et al., 2003), are, at the very least, needed to plan the action to be performed. The binding process seems to contribute to PM (Wang et al., 2010), associating the cue with the action(s) to be performed and the time at which it needs to be done. This contribution is assumed to be greater when individuals need to perform more than one action or when the link between the cue and the action to be performed is not obvious. Other factors that can influence PM functioning but do not necessarily underpin it, such as anxiety, are not represented in this figure. Of course, this summary figure will need to be confirmed in future research.

From a clinical perspective, these results shed new light on a possible source of anxiety, as TGA patients also have difficulty knowing what they are going to or have to do. It offers a possible explanation for verbal leitmotifs (Noël et al., 2008), as patients may retain residual knowledge about the fact that they have to do something in the immediate future (ProCom) but have virtually no recollection of what it is they have to do (RetCom), even if a third party gives them the information, as they will forget it again very quickly. The nature of these leitmotifs (i.e., "Have I fed the dogs?"; "Did I buy some bread?") is consistent with difficulty remembering what has to be done. In the same way, patients can perform actions that require $\mathrm{PM}$, in addition to procedural memory, like driving alone to some place for quite a long distance. Many patients in this situation keep driving to their destination, as they keep doing a task during the neuropsychological evaluation. 
Figure 3. PM functioning. The two-way arrows between the RetCom and ProCom underscore the close correlation between these two components of PM. Given this link, impairment of one component might well affect the other one too, even if this impairment was because of another cognitive function deficit (e.g., a massive episodic memory deficit might impact on the RetCom indirectly affecting the ProCom). In line with Umeda's hypothesis, this figure shows that the ProCom is linked to executive functions and the RetCom to episodic memory. Both ends of the episodic memory continuum are represented here: past (anterograde memory) and future (PM). Anterograde memory is thought directly to influence the RetCom of PM.

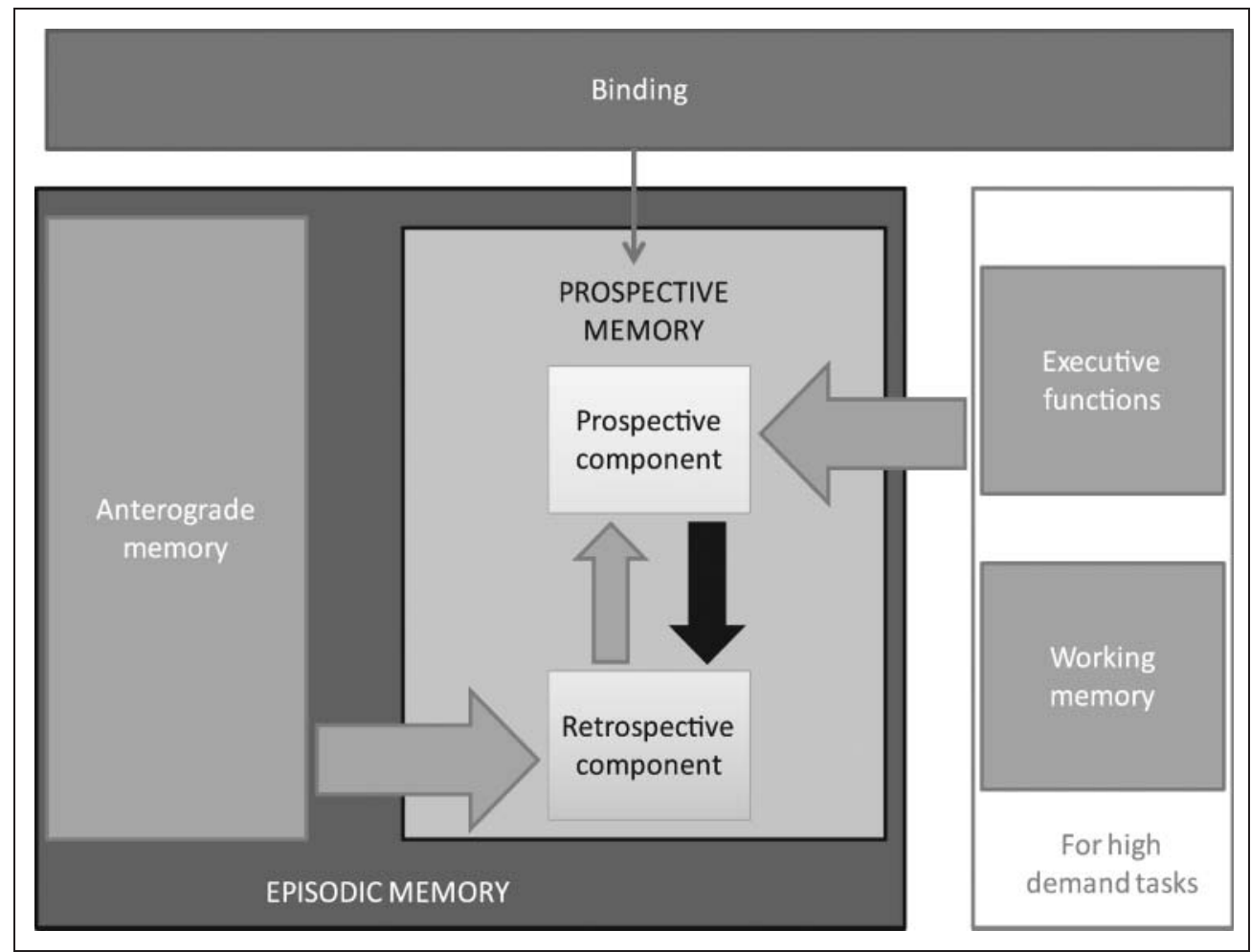

The role of action during TGA seems to be important in the residual memory abilities (including performing PM tasks) and leitmotifs during TGA and will need to be further explored.

This also reinforces the need to manage patients' and relatives' anxiety by providing verbal reassurance (Noël et al., 2008). TGA gives us a "scale model" of episodic memory and its time travel aspect, with patients having difficulty recalling the previous few minutes and remembering to do something in the immediate future, as though they were stuck in the present, and a similar pattern of recovery. This impaired mental travel is a possible cause of patients' perplexity during TGA, and further studies are needed to ascertain whether these patients can project themselves into the future and if so, how.

In conclusion, the present experiment demonstrated that TGA patients have difficulty remembering to perform an action in the future. By using a novel paradigm featuring two similar tasks, we were able to distinguish behaviorally between the ProCom and RetCom, although these two components of PM are closely connected. We discovered a strong link between anterograde memory and the RetCom, suggesting that PM could be part of episodic memory. From this perspective, executive functions may only be involved in the ProCom of PM and chiefly in the case of high-demand tasks, in line with the literature (Martin et al., 2003).

\section{APPENDIX: PATIENTS' AND CONTROLS' RESULTS}

Patients' and Controls' Results, Expressed in Mean Scores (SD) for Neuropsychological Measures

\begin{tabular}{|c|c|c|c|c|c|}
\hline & Acute Phase & Periacute Phase & Day After & Follow-up & Healthy Controls \\
\hline Orientation & $12.13(2.83)$ & $17.00(2.06)$ & $18.50(1.38)$ & $18.70(0.69)$ & $17.71(1.22)$ \\
\hline Semantic categorization (Mattis) & $6.33(1.40)$ & $6.88(1.93)$ & $7.60(1.10)$ & $7.09(0.95)$ & $7.86(0.52)$ \\
\hline Visuoconstructive abilities (Mattis) & $14.80(1.05)$ & $14.63(1.93)$ & $15.00(1.10)$ & $15.30(0.95)$ & $15.86(0.52)$ \\
\hline ProCom: correct responses & $7.00(3.79)$ & $8.70(3.09)$ & $9.45(1.04)$ & $9.82(0.73)$ & $9.86(0.53)$ \\
\hline RetCom: correct responses & $3.65(3.02)$ & $8.70(1.89)$ & $8.00(2.87)$ & $8.71(2.29)$ & $8.71(2.20)$ \\
\hline $\begin{array}{l}\text { ProCom: RT (msec) for } \\
\text { prospective items }\end{array}$ & $1107.02(696.25)$ & $1090.05(459.48)$ & $965.58(469.98)$ & $999.81(495.69)$ & 1269.11 (203.99) \\
\hline $\begin{array}{l}\text { ProCom: RT (msec) for } \\
\text { arithmetic items }\end{array}$ & $1520.07(440.52)$ & $1095.03(572.30)$ & $1107.81(598.80)$ & $1001.19(490.02)$ & $1339.76(286.76)$ \\
\hline
\end{tabular}




\begin{tabular}{lccccc}
\hline & Acute Phase & Periacute Phase & Day After & Follow-up & Healthy Controls \\
\hline $\begin{array}{l}\text { RetCom: RT (msec) for } \\
\text { prospective items }\end{array}$ & $1425.46(1256.91)$ & $1352.07(915.97)$ & $1629.22(1993.16)$ & $1144.61(781.31)$ & $1484.17(461.19)$ \\
$\begin{array}{l}\text { RetCom: RT (msec) for } \\
\quad \text { arithmetic items }\end{array}$ & $1583 .(523.69)$ & $972.04(570.08)$ & $1123.54(695.69)$ & $1013.48(513.99)$ & $1297.98(359.90)$ \\
ESR immediate cued recall & $13.05(2.53)$ & $14.90(0.88)$ & $15.72(0.40)$ & $15.84(0.44)$ & $15.86(0.36)$ \\
ESR free recall & $2.52(1.97)$ & $5.00(2.45)$ & $6.72(1.42)$ & $8.05(1.75)$ & $7.79(2.42)$ \\
ESR recognition & $11.29(4.41)$ & $15.30(1.57)$ & $16.00(0.00)$ & $15.50(2.45)$ & $15.93(0.27)$ \\
ESR \%R answers & $21.15(18.51)$ & $30.25(8.17)$ & $66.55(26.45)$ & $78.41(37.41)$ & $88.61(32.27)$ \\
ESR \%K answers & $53.54(21.12)$ & $67.18(18.91)$ & $32.18(10.58)$ & $18.11(5.64)$ & $10.54(5.23)$ \\
ESR \%G answers & $25.31(27.87)$ & $2.57(2.81)$ & $1.27(0.97)$ & $3.48(2.54)$ & $0.85(0.75)$ \\
TMT B: time & $103.76(43.87)$ & $96.9(33.72)$ & $110.36(50.90)$ & $85.95(37.69)$ & $89.07(57.27)$ \\
TMT B: errors & $0.52(0.59)$ & $0.50(0.55)$ & $0.81(0.79)$ & $0.34(0.38)$ & $0.33(0.35)$ \\
Stroop interference: time & $122.05(62.25)$ & $124.90(48.16)$ & $122.18(26.67)$ & $115.32(27.24)$ & $102.14(32.99)$ \\
Stroop interference: errors & $2.42(1.55)$ & $2.40(1.67)$ & $2.99(2.12)$ & $1.91(1.23)$ & $1.64(1.81)$ \\
\hline
\end{tabular}

Patients' and Controls' Results, Expressed in Mean Scores (SD) for Neuropsychological, Anxiety, and Mood Measures

\begin{tabular}{lccccc}
\hline Fluency task: correct responses & $17.29(3.93)$ & $19.60(0.78)$ & $19.84(0.64)$ & $19.89(0.32)$ & $19.95(0.21)$ \\
Fluency task: preservative errors & $1.76(1.64)$ & $0.40(0.97)$ & $0.09(0.30)$ & $0.05(0.22)$ & $0.36(0.99)$ \\
Forward digit span & $4.94(1.39)$ & $5.50(0.97)$ & $4.82(1.60)$ & $5.37(1.07)$ & $6.07(1.92)$ \\
Forward visuospatial span & $4.65(0.93)$ & $4.60(0.97)$ & $3.54(1.43)$ & $4.92(0.82)$ & $5.71(1.07)$ \\
Letter-number sequence task & $4.12(3.84)$ & $4.6(2.99)$ & $1.72(4.94)$ & $5.29(3.63)$ & $5.14(2.57)$ \\
Multimodal span task & $4.23(0.97)$ & $4.00(1.25)$ & $3.09(1.04)$ & $4.21(1.00)$ & $4.93(1.14)$ \\
Binding: accuracy & $4.06(6.60)$ & $4.2(6.19)$ & $7.18(4.53)$ & $9.34(6.40)$ & $9.86(5.12)$ \\
Binding: processing & $0.21(0.48)$ & $0.59(0.12)$ & $0.7(0.43)$ & $0.83(0.25)$ & $0.88(0.21)$ \\
BDI & $4.47(3.60)$ & $3.40(4.01)$ & $3.91(2.55)$ & $3.82(3.96)$ & $3.64(3.15)$ \\
Trait-STAI & $44.12(8.12)$ & $37.60(9.36)$ & $37.55(4.55)$ & $42.79(8.11)$ & $42.29(12.71)$ \\
State-STAI & $57.30(10.61)$ & $45.20(12.20)$ & $33.82(11.78)$ & $35.50(10.21)$ & $27.57(5.32)$ \\
Adjective mood scale & $24.47(11.60)$ & $18.70(11.73)$ & $16.18(9.08)$ & $15.56(8.67)$ & $8.07(6.43)$ \\
\hline
\end{tabular}

\%R answers = percentage of "Remember" answers, \%K answers = percentage of "Know" answers, \%G answers = percentage of "Guess" answers; TMT B = Part B of the TMT.

\section{Acknowledgments}

This work was supported by Caen University Hospital, as part of a clinical research project undertaken by Lower Normandy Regional Council, and by the Vicq d'Azyr Association, for the $\mathrm{PhD}$ funding of Mathieu Hainselin. The authors would like to thank Sandrine Bioux, Evangéline Bliaux, Carole Girard, Aurelija Juskenaite, and Guillaume Mazeure for collecting the data and Gregoria Kalpouzos, Julie Gonneaud, and Mickaël Laisney for their invaluable help and advice. We are also grateful to the staff of the Accident and Emergency Department, Neurology Units and Clinical Research Department at Caen University Hospital, and the Emergency Neurology Unit at Rouen University Hospital for their unstinting cooperation.
Reprint requests should be sent to Francis Eustache, Inserm-EPHEUniversité de Caen Basse Normandie, Unité 923, CHU Avenue de la Côte de Nacre, Caen, France, or via e-mail: neuropsycho@ chu-caen.fr.

\section{REFERENCES}

Adda, C. C., Castro, L. H., Alem-Mar e Silva, L. C., de Manreza, M. L., \& Kashiara, R. (2008). Prospective memory and mesial temporal epilepsy associated with hippocampal sclerosis. Neuropsychologia, 46, 1954-1964.

Baddeley, A. (2000). The episodic buffer: A new component of working memory? Trends in Cognitive Sciences, 4, 417-423. 
Bartsch, T., Alfke, K., Stingele, R., Rohr, A., Freitag-Wolf, S. Jansen, O., et al. (2006). Selective affection of hippocampal CA-1 neurons in patients with transient global amnesia without long-term sequelae. Brain: A Journal of Neurology, 129, 2874-2884.

Basso, D., Ferrari, M., \& Palladino, P. (2010). Prospective memory and working memory: Asymmetrical effects during frontal lobe TMS stimulation. Neuropsychologia, 48, 3282-3290.

Beck, A. T., Rial, W. Y., \& Rickets, K. (1974). Short form of Depression Inventory: Cross validation. Psychological Reports, 34, 1184-1186.

Blanco-Campal, A., Coen, R. F., Lawlor, B. A., Walsh, J. B., \& Burke, T. E. (2009). Detection of prospective memory deficits in mild cognitive impairment of suspected Alzheimer's disease etiology using a novel event-based prospective memory task. Journal of the International Neuropsychological Society, 15, 154-159.

Burgess, P. W., Quayle, A., \& Frith, C. D. (2001). Brain regions involved in prospective memory as determined by positron emission tomography. Neuropsychologia, 39, 545-555.

Burgess, P. W., Scott, S. K., \& Frith, C. D. (2003). The role of the rostral frontal cortex (area 10) in prospective memory: A lateral versus medial dissociation. Neuropsychologia, 41, 906-918.

Carlesimo, G. A., Casadio, P., \& Caltagirone, C. (2004). Prospective and retrospective components in the memory for actions to be performed in patients with severe closed-head injury. Journal of the International Neuropsychological Society, 10, 679-688.

Cohen, A. L., Dixon, R. A., Lindsay, D. S., \& Masson, M. E. (2003). The effect of perceptual distinctiveness on the prospective and retrospective components of prospective memory in young and old adults. Canadian Journal of Experimental Psychology, 57, 274-289.

Costa, A., Peppe, A., Caltagirone, C., \& Carlesimo, G. A. (2008). Prospective memory impairment in individuals with Parkinson's disease. Neuropsychology, 22, 283-292.

den Ouden, H. E., Frith, U., Frith, C., \& Blakemore, S. J. (2005). Thinking about intentions. Neuroimage, 28, 787-796.

d'Ydewalle, G., Bouckaert, D., \& Brunfaut, E. (2001). Age-related differences and complexity of ongoing activities in time- and event-based prospective memory. American Journal of Psychology, 114, 411-423.

Eichenbaum, H. (2001). The hippocampus and declarative memory: Cognitive mechanisms and neural codes. Behavioural Brain Research, 127, 199-207.

Einstein, G. O., \& McDaniel, M. A. (1990). Normal aging and prospective memory. Journal of Experimental Psychology: Learning, Memory, and Cognition, 16, 717-726.

Einstein, G. O., McDaniel, M. A., Richardson, S. L., Guynn, M. J., \& Cunfer, A. R. (1995). Aging and prospective memory: Examining the influences of self-initiated retrieval processes. Journal of Experimental Psychology: Learning, Memory, and Cognition, 21, 996-1007.

Eustache, F., \& Desgranges, B. (2008). MNESIS: Towards the integration of current multisystem models of memory. Neuropsychology Review, 18, 53-69.

Eustache, F., Desgranges, B., Laville, P., Guillery, B., Lalevée, C., Schaeffer, S., et al. (1999). Episodic memory in transient global amnesia: Encoding, storage, or retrieval deficit? Journal of Neurology, Neurosurgery and Psychiatry, 66, 148-154.

Godefroy, O. (2008). Fonctions exécutives et pathologies neurologiques et psychiatriques. Marseille: Solal.

Gonneaud, J., Kalpouzos, G., Bon, L., Vlader, F., Eustache, F., \& Desgranges, B. (2011). Distinct and shared cognitive functions mediate event and time-based prospective memory impairment in normal aging. Memory, 19, 360-377.

Greenberg, D. L., Keane, M. M., Ryan, L., \& Verfaellie, M. (2009). Impaired category fluency in medial temporal lobe amnesia: The role of episodic memory. Journal of Neuroscience, 29, 10900-10908.

Grober, E., \& Buschke, H. (1987). Genuine memory deficits in dementia. Developmental Neuropsychology, 3, 13-36.

Guillery, B., Desgranges, B., de La Sayette, V., Landeau, B., Eustache, F., \& Baron, J. C. (2002). Transient global amnesia: Concomitant episodic memory and positron emission tomography assessment in two additional patients. Neuroscience Letters, 325, 62-66.

Guillery, B., Desgranges, B., Katis, S., de La Sayette, V., Viader, F., \& Eustache, F. (2001). Semantic acquisition without memories: Evidence from transient global amnesia. NeuroReport, 12, 3865-3869.

Guillery, B., Desgranges, B., Piolino, P., Laville, P., de La Sayette, V., \& Eustache, F. (2000). Extensive temporally graded retrograde amnesia for personal-episodic facts in transient global amnesia. Neurocase, 6, 205-210.

Guillery-Girard, B., Desgranges, B., Urban, C., Piolino, P., de La Sayette, V., \& Eustache, F. (2004). The dynamic time course of memory recovery in transient global amnesia. Journal of Neurology, Neurosurgery and Psychiatry, 75, $1532-1540$

Henry, J. D., MacLeod, M. S., Phillips, L. H., \& Crawford, J. R. (2004). A meta-analytic review of prospective memory and aging. Psychology and Aging, 19, 27-39.

Hodges, J. R., \& Warlow, C. P. (1990). The aetiology of transient global amnesia. Brain: A Journal of Neurology, 113, 639-657.

Jäger, T., Bazner, H., Kliegel, M., Szabo, K., \& Hennerici, M. G. (2009). The transience and nature of cognitive impairments in transient global amnesia: A meta-analysis. Journal of Clinical and Experimental Neuropsychology, 31, 8-19.

Jones, S., Livner, A., \& Backman, L. (2006). Patterns of prospective and retrospective memory impairment in preclinical Alzheimer's disease. Neuropsychology, 20, $144-152$

Karantzoulis, S., Troyer, A. K., \& Rich, J. B. (2009). Prospective memory in amnestic mild cognitive impairment. Journal of the International Neuropsychological Society, 15, 407-415.

Kinsella, G. J., Ong, B., Storey, E., Wallace, J., \& Hester, R. (2007). Elaborated spaced-retrieval and prospective memory in mild Alzheimer's disease. Neuropsychological Rebabilitation, 17, 688-706.

Kliegel, M., \& Jager, T. (2006). The influence of negative emotions on prospective memory: A review and new data. International Journal of Computational Cognition, 4, 1-17.

Livner, A., Laukka, E. J., Karlsson, S., \& Backman, L. (2009). Prospective and retrospective memory in Alzheimer's disease and vascular dementia: Similar patterns of impairment. Journal of the Neurological Sciences, 283, 235-239.

Martin, M., Kliegel, M., \& McDaniel, M. A. (2003). The involvement of executive functions in prospective memory performance of adults. International Journal of Psychology, 38, 195-206.

Mattis, S. (1976). Mental status examination for organic mental syndrome in the elderly patient. In L. Bellack \& T. B. Karasu (Eds.), Geriatric psychiatry: A handbook for psychiatrics and primary care physicians (pp. 77-121). New York: Grune and Stratton.

McDaniel, M. A., \& Einstein, G. O. (2010). The neuropsychology of prospective memory in normal aging: A componential 
approach. Neuropsychologia. doi: 10.1016/j.neuropsychologia. 2010.12.029.

Noël, A., Quinette, P., Guillery-Girard, B., Dayan, J., Piolino, P., Marquis, S., et al. (2008). Psychopathological factors, memory disorders and transient global amnesia. British Journal of Psychiatry, 193, 1-7.

Northoff, G., \& Bermpohl, F. (2004). Cortical midline structures and the self. Trends in Cognitive Sciences, 8, 102-107.

Okuda, J., Fujii, T., Yamadori, A., Kawashima, R., Tsukiura, T., Fukatsu, R., et al. (1998). Participation of the prefrontal cortices in prospective memory: Evidence from a PET study in humans. Neuroscience Letters, 253, 127-130.

Quinette, P., Guillery, B., Desgranges, B., de La Sayette, V., Viader, F., \& Eustache, F. (2003). Working memory and executive functions in transient global amnesia. Brain: A Journal of Neurology, 126, 1917-1934.

Quinette, P., Guillery-Girard, B., Dayan, J., de La Sayette, V., Marquis, S., Viader, F., et al. (2006). What does transient global amnesia really mean? Review of the literature and thorough study of 142 cases. Brain: A Journal of Neurology, 129, 1640-1658.

Quinette, P., Guillery-Girard, B., Noël, A., de La Sayette, V., Viader, F., Desgranges, B., et al. (2006). The relationship between working memory and episodic memory disorders in transient global amnesia. Neuropsychologia, 44, $2508-2519$

Reynolds, J. R., West, R., \& Braver, T. (2009). Distinct neural circuits support transient and sustained processes in prospective memory and working memory. Cerebral Cortex, 19, 1208-1221.

Schacter, D. L., Addis, D. R., \& Buckner, R. L. (2007). Remembering the past to imagine the future: The prospective brain. Nature Reviews Neuroscience, 8 , 657-661.

Schacter, D. L., Addis, D. R., \& Buckner, R. L. (2008). Episodic simulation of future events: Concepts, data, and applications. Annals of the New York Academy of Sciences, 1124, 39-60.

Sedlaczek, O., Hirsch, J. G., Grips, E., Peters, C. N., Gass, A., Wohrle, J., et al. (2004). Detection of delayed focal MR changes in the lateral hippocampus in transient global amnesia. Neurology, 62, 2165-2170.

Signoret, J. L. (1991). Batterie d'efficience mnésique: BEM 144. Amsterdam: Elsevier.
Signoret, J. L., Allard, M., Benoit, N., Bolgert, F., Bonvarlet, M., \& Eustache, F. (1989). Evaluation des troubles de mémoire et des désordres cognitifs associés, BEC 96. Paris: Fondation IPSEN.

Spielberger, C. D. (1983). Manual for the State-Trait Anxiety. Palo Alto, CA: Consulting Psychologist Press.

Szpunar, K. K., Chan, J. C., \& McDermott, K. B. (2009). Contextual processing in episodic future thought. Cerebral Cortex, 19, 1539-1548.

Thompson, C., Henry, J. D., Rendell, P. G., Withall, A., \& Brodaty, H. (2010). Prospective memory function in mild cognitive impairment and early dementia. Journal of the International Neuropsychological Society, 16, 318-325.

Tulving, E. (2002). Episodic memory: From mind to brain. Annual Review of Psychology, 53, 1-25.

Umeda, S., Naqumo, Y., \& Kato, M. (2006). Dissociative contributions of medial temporal and frontal regions to prospective remembering. Reviews in the Neurosciences, 17, 267-278

Uttl, B. (2008). Transparent meta-analysis of prospective memory and aging. PloS ONE, 3, e1568.

Viard, A., Chételat, G., Lebreton, K., Desgranges, B., Landeau, B., de La Sayette, V., et al. (2011). Mental time travel into the past and the future in healthy aged adults: An fMRI study. Brain and Cognition, 75, 1-9.

Von Zerssen, D., Koeller, D. M., \& Rey, E. R. (1970). A scale for the objective evaluation of the state of subjective well-being as a method for longitudinal studies. Arzneimittel-Forschung, 20, 915-918.

Wang, W., Dew, I., \& Giovanello, K. (2010). Effects of aging and prospective memory on recognition of item and associative information. Psychology and Aging, 25, 486-491.

Wechsler, D. (1969). Echelle clinique de mémoire (forme 1). Paris: Editions du Centre de Psychologie Appliquée.

Wechsler, D. (1991). Echelle clinique de mémoire révisée. Paris: Editions du Centre de Psychologie Appliquée.

Wechsler, D. (1997). Wechsler Memory Scale-III. San Antonio, TX: The Psychological Corporation.

West, R., \& Krompinger, J. (2005). Neural correlates of prospective and retrospective memory. Neuropsychologia, 43, 418-433.

Wheeler, M. A., Stuss, D. T., \& Tulving, E. (1997). Toward a theory of episodic memory: The frontal lobes and autonoetic consciousness. Psychological Bulletin, 121, 331-354. 